\title{
Impact of generator start-up lead times on short-term scheduling with high shares of renewables
}

\author{
Mathias Hermans ${ }^{a, b}$, Kenneth Bruninx $x^{a, b, c}$ and Erik Delarue ${ }^{a, b, *}$ \\ ${ }^{a}$ KU Leuven, Celestijnenlaan 300, 3001 Leuven, Belgium \\ ${ }^{\mathrm{b}}$ EnergyVille, Thor Park 8310, 3600 Genk, Belgium \\ ' VITO, Boeretang 200, $2400 \mathrm{Mol}$, Belgium \\ *Corresponding author: erik.delarue@kuleuven.be ; +32 16322521
}

\begin{abstract}
To cope with the variability and uncertainty introduced by, i.a., intermittent renewable energy sources, the flexible planning and operation of generation units is crucial. Their reaction time is constrained by the lead times on the start-up decisions, whereas the demand for flexibility and operating reserves depends on the market clearing frequency. The start-up lead times are limited by the operator's tolerance for increased maintenance on the asset, which should be reflected in short-term scheduling models. To study this interaction between the market clearing frequency and the start-up capabilities of combined-cycle gas turbines, we develop a unit commitment model. The model considers multiple start-up trajectories and the scheduling decisions in joint energy-operating reserve and balancing markets. The uncertainty on wind power forecasts is presented via wind power forecast updates generated by a dedicated data-driven tool. Leveraging this model, we investigate the interaction between (i) the frequency of wind power forecast updates, linked to the market clearing frequency, (ii) cost-optimal operating reserve volumes and (iii) combined-cycle gas turbine start-up decisions. Results show that, in general, higher market clearing frequencies lead to lower operating costs, driven by decreasing volumes of operating reserves and facilitated by the fast start-up capabilities of combined-cycle gas turbines.
\end{abstract}

Keywords - Power plant flexibility, balancing, operating reserves, uncertainty, combined-cycle gas turbine, start-up trajectories, unit commitment

\section{Introduction}

In power systems with high shares of variable and limitedly predictable renewable energy sources (VRES), 
operating reserve requirements are increasingly driven by the uncertainty on VRES forecasts. Their accuracy, however, improves significantly as the time between forecast creation and actual realization decreases. Making use of forecasts with shorter lead times to frequently adjust the operating schedule would reduce the real-time operating reserve requirements and could yield operational cost savings while maintaining the system's reliability [1]. Bruninx and Delarue [2] underline the importance of using an accurate statistical description of forecast errors when dimensioning reserve requirements in power systems with high shares of vRES.

An important potential for taking advantage of improved forecasting closer to the time of delivery is situated after the day-ahead market clearing (12 to 36 hours before delivery) $[46,47,48]$. This allows for uncertainty to have an important impact on the system. However, intraday optimization processes and markets can unlock the potential for recourse decisions when generation companies' forecasts change. Recourse decisions usually involve changes to the previously scheduled economic dispatch of generation units, but could also affect the unit commitment decisions, i.e., involve shut-downs or start-ups. Such alterations should evidently conform to the limitations of power plant flexibility. Flexibility provided by the dynamic operation of thermal electricity generation units can be split up into two aspects. First, residual load variations frequently require considerable ramping of generation units. Second, if modulating the already committed units will not suffice, additional units start up, providing flexibility in the form of the start-up loading ramp rate. Changing the scheduled output of online units may induce increased wear-and-tear, but is typically not limited by the technical ramping constraints of, e.g., flexible combined-cycle gas turbines. Bringing additional units online, however, can be challenging. To prepare the unit for start-up loading, sufficient time must be available, which depends on the offline time and the operator's tolerance for increased maintenance on the asset. The start-up procedures may take considerable time and entail significant operating costs. The shorter the start-up lead time, the more easily a generation unit can accommodate unexpected flexibility needs, which demonstrates the importance of accurate modeling of start-up time constraints [3].

The focus of this paper is therefore on the impact of start-up lead times in a unit commitment and economic dispatch (UC/ED) model, taking a rolling horizon approach to capture wind power uncertainty and the functioning of joint energy-operating reserve and balancing markets. This model performs a central economic dispatch under the assumption of perfect competition, mimicking most US markets [4] and a few European markets (e.g. Greece, Hungary, Ireland, Italy, Northern Ireland and Poland). Its outcome is very similar to a market design where electricity is traded day-ahead bilaterally and through 
power exchanges, as in other European countries $[5,6]$.

In the literature, several UC models have been proposed to represent and deal with vRES uncertainty. Some authors have developed deterministic UC models (DUC) [7-10], while others have focused on stochastic UC models (SUC) [1,11-16]. SUC implementations typically lead to more cost-efficient schedules compared to their DUC equivalents $[15,16]$. However, SUC models typically require a large number of scenarios to generate meaningful solutions and the computational cost may strongly increase with the number of scenarios one considers $[17,18]$. Although this issue may be mitigated by dedicated scenario reduction techniques [19] and/or decomposition and parallelization [20], the quality of the solution remains dependent on the quality of the scenario set [19]. To avoid these drawbacks, Bruninx and Delarue [17] have developed a state-of-the-art DUC model with probabilistic reserve requirements (DUC-PRR), yielding UC schedules that are nearly as cost-effective as those obtained from an equivalent SUC problem, at a computational cost comparable to that of a DUC problem.

Several authors studied how the flexibility of gas-fired generation units may be presented in such UC models. Prina et al. [49], e.g., present the integration of combined cycle gas turbine (CCGT) flexibility constraints and cycling costs into the EPLANopt model [50], and compare several studies that address the impact of operational flexibility in energy system modeling, of which most comprise some type of unit commitment model. This literature review focusses on unit commitment models taking a rolling horizon approach to to study the attainable cost savings associated with recourse decisions assuming varying market clearing frequencies. Table I gives an overview of the various approaches taken in these studies to enforce start-up decision lead times when making recourse decisions that affect the unit commitment schedule. Tuohy et al. [1] and Meibom et al. [14] apply the Wilmar SUC model to study the effects of high levels of stochastic wind power and electricity demand on thermal power plant scheduling performance. Start-up lead times are accounted for in an aggregated way, by fixing the total online capacity of groups of units in the first hours of the rolling planning horizon to the online capacity scheduled for the corresponding period in the previous planning loop. The rolling planning approaches taken in Hermans et al. $[7,45]$ and Córdova et al. [39] impose no restrictions on the scheduling of new start-ups close to the moment of delivery. Conversely, Jafari et al. [13] restrict all deviations from the day-ahead commitments (i.e., start-ups and shut-downs) when investigating the value of intra-day markets to optimize daily generation scheduling in power systems with high wind power penetration. Finally, Schulze and Mckinnon [16] implement non-anticipativity constraints to link start-up decisions across scenario bundles in a SUC model, but do not restrict changes to the start-up decisions themselves when updating the scenario tree 
and generation schedule. As shown in Table I, none of these works considered a detailed representation of generation unit-specific start-up lead times, being differentiated by their dependence on the boiler temperature state (hot, warm or cold) and the trade-off between increased maintenance on the asset and faster start-up procedures.

\begin{tabular}{|c|c|c|c|c|}
\hline Authors & $\begin{array}{l}\text { Model } \\
\text { type }\end{array}$ & $\begin{array}{l}\text { Temporal } \\
\text { resolution }\end{array}$ & $\begin{array}{l}\text { Constraints related to start-up lead } \\
\text { times }\end{array}$ & $\begin{array}{l}\text { Temperature dependent } \\
\text { start-up considered }\end{array}$ \\
\hline Tuohy et al. [1] & SUC & Hourly & $\begin{array}{l}\text { Online capacity of unit group fixed in } \\
\text { first lead time hours }\end{array}$ & No \\
\hline Hermans et al. [7] & DUC & Hourly & Unrestricted & $\begin{array}{l}\text { Hot, warm, cold start-up } \\
\text { costs }\end{array}$ \\
\hline Jafari et al. [13] & SUC & Hourly & $\begin{array}{l}\text { Start-up and shut-down decisions fixed } \\
\text { to day-ahead schedule }\end{array}$ & No \\
\hline Meibom et al. [14] & SUC & Hourly & $\begin{array}{l}\text { Online capacity of unit group fixed in } \\
\text { first lead time hours }\end{array}$ & No \\
\hline $\begin{array}{l}\text { Schulze and } \\
\text { Mckinnon [16] }\end{array}$ & SUC & Hourly & $\begin{array}{l}\text { Non-anticipativity constraints converge } \\
\text { start-up decisions across scenario } \\
\text { bundles within notification period }\end{array}$ & No \\
\hline Córdova et al. [39] & CIUC & Hourly & Unrestricted & No \\
\hline Hermans et al. [45] & DUC & Quarter-hourly & Unrestricted & No \\
\hline Present work & DUC-PRR & Quarter-hourly & $\begin{array}{l}\text { Generation unit-specific minimum } \\
\text { start-up decision lead times, with } \\
\text { choice for faster start-up procedure }\end{array}$ & $\begin{array}{l}\text { Hot, warm, cold } \\
\text { differentiation of start- } \\
\text { up lead times and costs }\end{array}$ \\
\hline
\end{tabular}

Table I: Literature overview of the approaches taken in the literature to impose minimum start-up decision lead times when making recourse decisions.

In [21], we extended a state-of-the-art DUC formulation [22] to include multiple start-up loading ramp rates of CCGT units and their associated maintenance costs. Leveraging an extended version of the model in [21], we analyzed the relation between the provision of spinning reserve capacity and CCGT start-up decisions for various shares of vRES and dynamic reserve requirements in [7]. Faster but more costly startups were found to reduce the total operating costs and the cost-optimal reserve requirements. The work in $[7,21]$, however, focused exclusively on the increased flexibility provided by increased start-up loading ramp rates, and did not consider the duration of the start-up procedure prior to loading, nor did it account for this duration as a minimum lead time when making or adjusting scheduling decisions based on updates of renewable energy forecasts. Furthermore, reserve requirements were imposed exogenously, whereas in what follows, we adopt probabilistic reserve constraints, which facilitate online, cost-optimal reserve sizing [17].

In a rolling planning approach, the model should be provided with updated forecasts to which the scheduling decisions are adjusted. In a statistical analysis of observed wind power forecast errors, detailed in Section 2.2, we found a significant correlation between the errors of day-ahead and intra-day forecast 
updates. The wind power forecast updates employed in our rolling horizon approach should be consistent with this observation. Some techniques account for inter-temporal correlations to reflect historical forecast variability along the look ahead period, as proposed by Pinson et al. [40] and refined by Ma et al. [41]. However, to the best of the authors' knowledge, the correlation of point forecast errors between consecutive forecast updates has not been considered in the literature.

In summary, this work contributes to the existing literature in the following ways:

1) We combine a state-of-the-art UC model [22] with probabilistic reserve constraints [17] and a representation of multiple start-up modes, differentiated by the required lead time and associated maintenance costs. Start-up costs and start-up lead times in turn depend on the boiler temperature of the power plant. The approach taken in this work allows capturing the constraints introduced startup times in detail (see Table I). Alternative formulations have been proposed that aim to very accurately present power trajectories at the level of a single unit, such as in $[42,43]$, depending on the specific CCGT assembly (i.e., the number of gas and steam turbines) of the unit. This article, however, addresses the duration of the start-up procedure prior to loading as a minimum lead time when making or adjusting scheduling decisions. Therefore, such formulations that model the possible transitions between gas and steam turbines configurations were deemed to be too computationally demanding for the purpose of this study. We apply the resulting model in a three-stage rolling horizon approach that captures the functioning of a joint energy-operating reserve and balancing markets as in $[1,7,13,14,16,39]$.

2) We present a dedicated data-driven tool to generate wind power forecast updates that mimic the accuracy of today's forecasting tools. The accuracy of these forecasts, e.g., improves as the forecast lead time decreases. The forecast generation tool reflects the correlation of point forecast errors between historically observed wind power forecast updates. As such, it is fit to be used in a rolling planning DUC model with reserve constraints that carefully accounts for power plant start-up times.

3) We apply the resulting framework in a case study to highlight the interaction between a higher market clearing frequency, which enables using more accurate vRES forecasts and carrying less operating reserve capacity, and the start-up lead times of CCGT units, taking into account peaking units as a competing source of flexibility. As opposed to the hourly time resolution adopted in $[1,7,13,14,16,39]$, a quarter-hourly time resolution is employed to adequately capture the flexibility needs of the system and the activation of balancing reserves, following $[8-10,15]$ and as recommended by FERC for high 
VRES systems [23]. The robustness of the results towards our assumptions on the marginal production cost of the peaking units; the share of renewables and the flexibility of the rest of the power system is examined in a sensitivity analysis.

The results of our case studies reveal a total operating cost decrease up to $1.9-2.3 \%$ with high market clearing frequencies, driven by decreased wind power uncertainty with lower forecasting lead times. We show, however, that one should take caution when increasing the market clearing frequency, as very frequently adjusting the production schedule to the most recent wind power forecast update may lead to higher operational costs due to possible biases in the production schedule toward erroneous forecasts and interactions with the start-up lead time. Higher market clearing frequencies make the need for fast CCGT starts more apparent, despite their increased maintenance costs. Finally, we find that in high vRES power systems, the cost reduction obtained by a shift towards sub-daily planning is greatest for power systems with a high share of inflexible capacity and relatively few gas-fired power plants.

This paper proceeds as follows. Section 2 presents the developed methodology, the wind power forecast scenario generation tool and the test system for the case study. Section 3 presents and discusses the simulation results. Last, Section 4 concludes.

\section{Methodology}

To model joint energy-reserve and balancing markets, a rolling horizon approach is taken as illustrated in Fig. 1. The market clearing frequency is represented by splitting the total simulation period into overlapping planning loops, which allows adjusting the unit commitment schedule as the model is provided with updated wind power forecasts. Each planning loop has a horizon which consists of a balancing stage $A$, a final commitment stage $B$ (representing a joint energy-reserve market clearing period and setting the market clearing frequency) and an update stage $C$ (Fig. 1). The commitment statuses in stage $B$ and $C$ are allowed to be adjusted with respect to the previous loop, as long as all technical limitations, including the start-up lead times, are respected. The schedule in stages $B$ and $C$ is subject to reserve requirements and wind power forecast errors, representing the day-ahead and intra-day markets. At the first time step of the final commitment stage $B$, the scheduling decisions are subject to real time wind power uncertainty. The forecast lead times (and the associated uncertainty) increase along the rest of the horizon. Finally, the commitment decisions made in stage $B$ are carried over to the balancing stage (A) of the next market clearing, where they are fixed. The forecast in the balancing stage $(A)$ is assumed 
to be perfect and the reserve requirements are lifted, representing the activation of the procured reserves in the balancing market.

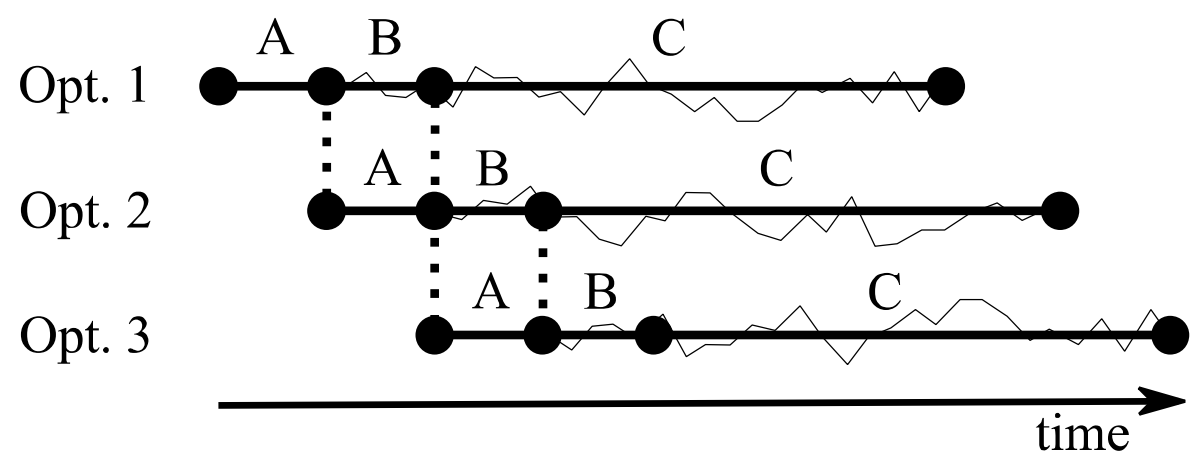

Fig. 1. The planning horizon of each optimization (market clearing) consists of a balancing stage A, a final commitment stage $B$ and an update stage $C$. As opposed to the balancing stage $A$, the commitment statuses in stage $B$ and $C$ are allowed to be adjusted to the most recent wind power forecast (curved line). (Figure reused from [7].)

Below, we first discuss the employed unit commitment model. Second, the wind power forecast scenario generation tool is presented and validated. Finally, we describe the test system and Monte Carlo approach taken for the case studies.

\subsection{Unit commitment model description}

We briefly present the deterministic unit commitment model [22], extended with probabilistic reserve constraints [17], that enables multiple start-up modes, formulated as a mixed integer linear program. This model allows accounting for the cost-optimal sizing, allocation and expected activation cost of reserve providers, which leads to more cost-optimal UC schedules than can be obtained from typical deterministic models. This is enabled by complementing the operating cost under forecast conditions (fuel costs $c_{i, j}^{F}$, start-up $\operatorname{costs} c_{i, t}^{S U}$, ramping $\operatorname{costs} c_{i, t}^{R}, \operatorname{cO} 2$-emission $\operatorname{costs} c_{i, t}^{C O 2}$ and the cost of load shedding $\lambda^{\Phi} \cdot \Phi_{t}$ ) with the expected cost of activating reserves in the commitment stage $B$ and update stage $C$ (Fig. 1):

$$
\begin{aligned}
& \min \sum_{t}\left(\sum_{i}\left(c_{i, t}^{S U}+c_{i, t}^{F}+c_{i, t}^{C O 2}+c_{i, t}^{R}\right)+\lambda^{\Phi} \cdot \Phi_{t}\right)+\sum_{t} \sum_{l}\left(P_{t, l}^{+}\left(\sum_{i} a c_{i, t, l}^{R+}+a c_{i, t, l}^{N S R+}+\lambda^{\Phi} \cdot \Phi_{t, l}^{L+}\right)+\right. \\
& \left.\quad P_{t, l}^{-} \sum_{i} a c_{i, t, l}^{R-}\right)
\end{aligned}
$$

These activation costs are determined by the operating costs associated with each flexibility option scheduled to provide the reserves in a reserve level $l \in L$ (spinning reserves $a c_{i, t, l}^{R+}$ and $a c_{i, t, l}^{R-}$, non-spinning reserves $a c_{i, t, l}^{N S R+}$ or load-shedding $\lambda^{\Phi} \cdot \Phi_{t, l}^{L+}$, see below), and weighted by the activation probability of the reserve level / (upward: $P_{t, l}^{+}$, downward: $P_{t, l}^{-}$) [17]. Spinning and non-spinning reserves result in fuel and CO2-emission costs. Activating upward reserves results in an increase in operating costs $\left(a c_{i, t, l}^{R+}, a c_{i, t, l}^{N S R+} \geq\right.$ 
0 ), while activating downward reserves will trigger cost reductions $\left(a c_{i, t, l}^{R-} \leq 0\right)$ as fuel is saved. The activation of curtailment of RES-based generation as downward reserve provider is assumed to be available at zero cost, while the expected volume of additional real-time load shedding due to scheduling load shedding as upward reserve provider $\left(P_{t, l}^{+} \cdot \Phi_{t, l}^{L+}\right)$ is penalized at the value of lost load (VOLL, $\left.\lambda^{\Phi}\right)$.

At each time step $t \in T$, the supply of electricity by conventional power plants $i \in I\left(g_{i, t}\right)$ and renewable energy sources $\left(G_{t}^{F}\right)$, corrected for curtailment $\left(\chi_{t}\right)$ must match the demand for electricity $\left(D_{t}\right)$ minus load curtailment $\left(\Phi_{t}\right)$ under forecast conditions:

$\forall j: D_{t}-\Phi_{t}=\sum_{i} g_{i, t}+G_{t}^{F}-\chi_{t}$

In what follows, we first briefly discuss the operating reserve requirements, based on [17]. Second, we present novel technical constraints needed to enforce the start-up decision lead times. Other technical constraints, such as binary logic constraints, generation limits, start-up loading (SU) and upward/downward ramping limits (RU/RD) and minimum up and down time (MUT/MDT) constraints are not discussed for sake of brevity. A full description of these constraints can be found in $[22,18]$.

\subsubsection{Probabilistic operating reserve requirements}

The upward and downward reserve requirements $D_{t, l}^{+}$and $D_{t, l}^{-}$are split in a number of discrete intervals L, creating reserve levels I, each of which correspond to a specific activation probability, following [17]. The statistical analysis of the wind power uncertainty, which is assumed to be the only source of uncertainty and further detailed in Section 2.2, yields probability density functions of the forecast errors from which the activation probabilities of each reserve level at every time step can be calculated following Bruninx and Delarue [17]. At each time step, the upward and downward reserve requirement must be satisfied:

$\forall t, \forall l: \quad D_{t, l}^{+}=\sum_{i} r_{i, t, l}^{L+}+n s r_{i, t, l}^{L+}+\chi_{t, l}^{L+}+\Phi_{t, l}^{L+}$

$\forall j, \forall l: \quad D_{t, l}^{-}=\sum_{i} r_{i, t, l}^{L-}+\chi_{t, l}^{L-}$

Aside from spinning $\left(r_{i, t, l}^{L+}\right.$ and $\left.r_{i, t, l}^{L-}\right)$ or non-spinning reserves $\left(n s r_{i, t, l}^{L+}\right)$ provided by conventional generation units, additional load shedding $\left(\Phi_{t, l}^{L+} \geq 0\right)$ and curtailment of RES-based generation $\left(\chi_{t, l}^{L-}, \chi_{t, l}^{L+} \geq 0\right)$ are explicitly considered as flexibility options. RES-based generation as upward reserve provider is limited to the scheduled curtailment of the forecasted wind and solar power $\left(\sum_{l} \chi_{t, l}^{L+} \leq \chi_{t} \leq G_{t}^{F}\right)$. As such, the reserve sizing problem has been internalized in the UC problem [17]. 


\subsubsection{Start-up lead times of CCGT units}

Start-up decisions made or changed in each market clearing are constrained by the time needed to prepare the unit for start-up loading. Firstly, the start-up decision lead time $S U T_{i, t, m}$ depends on the boiler temperature state of the generation unit $b \in\{$ hot, warm or cold $\}$ at the envisioned time of start-up $t$, which is accounted for as in [24]. Note, however, that for each unit $i$, the temperature state $b$ corresponding to time step $t$ can be determined from the preceding market clearing outcomes and, hence, can used to build $S U T_{i, t, m}$ during pre-processing between optimizations. Therefore, no index $b$ is necessary. Secondly, $S U T_{i, t, m}$ depends on the chosen start-up mode $m$, if multiple options are allowed. Evidently, any newly made decision $v_{i, t, m, b}$ to start up a generation unit in mode $m \in M$ and temperature state $b$ at time step $t$ should respect the appropriate start-up lead time $S U T_{i, t, m}$. Confirming a decision to start-up a unit at time $t$ in mode $m$ that was already made in a previous market clearing is not constrained by start-up lead time restrictions, nor is any deferral of that start-up to a later time. However, if a start-up is moved up to an earlier time, that decision should respect the start-up lead time restrictions. Any part of the start-up procedure that has been completed in the time between planning updates, following the earlier decisions, is then subtracted from $S U T_{i, t, m}$ for the subsequent market clearing. The following constraint then ensures that all minimum lead time restrictions are respected:

$\forall i \in I, t \in\left[T^{\text {balancing }}+1, \ldots, T^{\text {balancing }}+S U T_{i, t, m}\right], m \in\{$ standard or fast $\}:$

if $\quad \sum_{t^{\prime}=T^{\text {balancing }_{+1}}}^{t^{\prime}=t} V F_{i, t, \boldsymbol{m}}=0$

then $\sum_{b} v_{i, t, m, b}=0$

$T^{\text {balancing }}$ is the length of the balancing stage (Fig. 1), which is also the market clearing frequency in all our simulations. Note that the start-up lead time restrictions apply up to time step $T^{\text {balancing }}+S U T_{i, t, m}$, since the beginning of stage $B$ represents the moment at which the market is cleared (see Fig. 1). $V F_{i, t, m}$ are the start-up decisions for the commitment stage $B$ and the adjustment stage $C$ (Fig. 1) made in the preceding market clearing. At time step $t$ and for start-up mode $m$, one of the following statements holds for any unit i:

1) $\sum_{t^{\prime}=1}^{t^{\prime}=t} V F_{i, t \prime, m}>0$, which means that a start-up was already planned in period $T^{\prime}=\left[T^{\text {balancing }}+\right.$ $1, \ldots, t]$. If it was planned for time step $t$, this start-up decision can either be reconfirmed or delayed, since the start-up restrictions have already been satisfied in the preceding market clearing. If it was planned for an earlier time step, an additional start-up can be planned at time step $t$, on the 
condition that the MUT/MDT constraints are not binding. The MUT/MDT constraints ensure that (i) when a unit is started, it remains online for at least a certain amount of hours and (ii) when a unit is stopped, it remains offline for at least a certain amount of hours. Although they interact with the constraint enforcing the start-up lead times (Eq. (5)), they do not have the same goal - they are complementary. While Eq. (5) restricts decisions about the first start-up on the scheduling horizon, the MUT/MDT constraints (of which a full description can be found in $[22,18]$ ) foresee enough time between consecutive start-ups.

2) $\sum_{t^{\prime}=1}^{t^{\prime}=t} V F_{i, t, m}=0$, which means that a decision to start up would be new, or that a start-up initially planned for time step $t^{\prime \prime}>t$ is moved up (and possibly changed to a faster mode $m$ ). Either decision should respect the start-up lead time restrictions (5).

\subsection{Wind Power Forecast Scenario Generation for rolling planning unit commitment}

In UC model applications that do not involve rolling planning, the forecast typically originates from publicly or privately available historical forecast data [18]. However, given the limited availability of historical forecast updates with suitable parameters for use in rolling horizon UC models (e.g., update frequency and horizon), their origin should be a point of attention. Indeed, if actual forecasts are not available, they must themselves originate from a forecast scenario generation tool that mimics the behavior of the forecasting tool employed in the system at hand.

Deploying the rolling planning methodology requires a careful approach to forecast scenario generation. By definition, the forecast scenario updates overlap (Fig. 1), and the relative position of the forecast scenarios to the actual realization affects the resulting UC schedule, even if the procured flexibility (e.g., in the form of reserve requirements) adequately captures the uncertainty. Therefore, it is a cause for concern if the forecast updates (around which explicit reserve requirements can be imposed) do not exhibit a realistic correlation between them. Indeed, the existence of a positive correlation between the errors of forecasts made for the same target time, but with different lead times is supported via a statistical analysis presented below.

Especially when the availability of recourse decisions is subject to realistic limitations (e.g., start-up times), the scenario generation tool should adhere to the observed correlations when generating forecast updates. If not, the schedule is at risk of randomly (i.e., without correlation) having to be adjusted through recourse decisions. Recourse decisions may not be reversible (e.g., postponing start-ups or shutting units down for the minimum down time) or may only be reversible at additional costs (e.g., through shorter, 
but more costly, start-up procedures, by starting up other generation units with greater start-up or generation costs, or by violating spinning reserve requirements). In the context of this work, neglecting this critical feature of the used forecast scenario generation tool could lead to less cost-efficient or less reliable generation schedules and inaccurate estimations of the need for flexible power plant operation.

Below, we present a forecast scenario generation technique that aims to carefully mimic the historically observed correlations between forecasts that are regenerated (i.e., updated) with decreasing lead times, as well as the accuracy of today's forecasting tools.

Day-ahead and intra-day wind power forecast and measured power data with a quarter-hourly resolution from January 2016 to December 2018 for Belgium were obtained from Elia, the Belgian TSO [25]. In a statistical analysis, we observed three characteristics of wind power forecast errors that should be reflected in the generated wind power forecasts:

1) Wind power forecast tools perform better as the time between forecast creation and actual realization (i.e., the lead time) decreases (Fig. 3);

2) The measured power and the forecast error in the previous (quarter-hourly) time step were found to impact the statistical characteristics of the observed forecast errors, which are limited to a range around the forecast error in this previous time step;

3) A significant correlation between the available day-ahead and intra-day forecasts was observed.

Therefore, we calculate the probability density function (PDF) and the cumulative probability density function (CDF) of the historically observed forecast errors, for each observed combination of lead time, a predetermined set of measured power (MP) intervals and intervals of the forecast error in the previous time step (PFE, which correspondingly has a lead time reduced by one qh). Prior to analysis, the time series and forecast errors were normalized with respect to the available wind power capacity. To obtain an adequate resolution while avoiding over-fitting of the data, the observed forecast errors were each assigned to one combination of one of $4 \mathrm{MP}$ and one of 40 PFE intervals of equal size, yielding $4 \times 40$ distributions for each of 148 lead times (resolution: $1 \mathrm{qh}$ ). Histograms were set up by splitting the total range of possible forecast errors $([-1,+1])$ into equal power bins with a resolution of 0.01 . Each forecast error was then allocated to its corresponding error power bin. Finally, all 148 sets of $4 \times 40$ histograms were converted to the corresponding $4 \times 40$ PDFs and CDFs. In addition, we calculate a correlation matrix $\Sigma^{h}$ for all observed combinations of lead times between the two time series. 
Forecasts are generated as disturbances (errors) to the measured wind power (historical data). The forecast generation method samples from the PDFs of forecast errors as follows. First, for the target forecast time, a vector $\mathrm{N}\left(0, \Sigma^{c}\right)$ of random realizations of the standard multivariate normal distribution (SND) is generated, representing normalized forecasts with lead times of 1 to $148 \mathrm{qh}$. The input correlation matrix $\Sigma^{c}$ ensures the interdependence of the realizations with respect to their lead times. Second, the normally distributed realizations are transformed to wind power forecast error scenarios via the CDFs acquired in the statistical analysis, following [18]. The inverse CDF associated with the lead time of each separate sample, the MP power bin at the target time and the PFE power bin is evaluated at the cumulative probability of the respective random sample of the standard multivariate normal distribution. This process is repeated for each time step of the simulation period. Finally, the corresponding measured wind power is added to the resulting vectors of forecast errors to obtain the forecasts $F_{l t=1 \ldots 148}^{t=1 \ldots T}$. By concatenating forecasts of consecutive target and lead times $\left(\left[F_{l t=1}^{t=1}, F_{l t=2}^{t=2}, \ldots, F_{l t=\text { forecast horizon }}^{t=\text { forecast }}\right]\right)$, the desired wind power forecast updates can be selected. As the imposed correlation between the standard normally distributed samples $\Sigma^{c}$ is not preserved through the transformation to wind power forecast errors, an iterative approach was taken to adjust the input correlation matrix $\Sigma^{c}$ until the lead time correlation matrix for a full year of generated forecast errors approximated the historical correlation matrix $\Sigma^{h}$ as close as possible.

A series of six-hourly forecast updates obtained from the scenario generation technique is plotted in Fig. 2. Figure 3 shows that the mean absolute percentage error (MAPE) in function of the forecast lead time for one year of generated forecasts (forecast update frequency: $1 \mathrm{qh}$ ) approximates that of historical forecast data. Note the increasing inaccuracy of the forecasts with the lead time of the forecast. The quantiles of the historical forecasts, as a measure of the spread and distribution of the forecast errors in function of the forecast lead time, approximate those of the generated forecasts for the same period (Fig. 4). Finally, we found a root mean squared error (RMSE) of 0.158 (16.8\%) between the correlation coefficients observed in historical and generated forecast data with lead times of 24 hours or less. Hence, the forecast generation tool reflects the historical correlation of point forecast errors between consecutive forecast updates. We stress again that this last feature is crucial in this work, where the feasibility of bringing more units online as part of recourse decisions is subject to start-up lead times. As such, the generated forecasts comply with the three observed characteristics discussed above. These properties make the forecasting scenario generation tool suitable for studying the impact of generator start-up times on short-term scheduling and associated costs with high shares of renewables. 


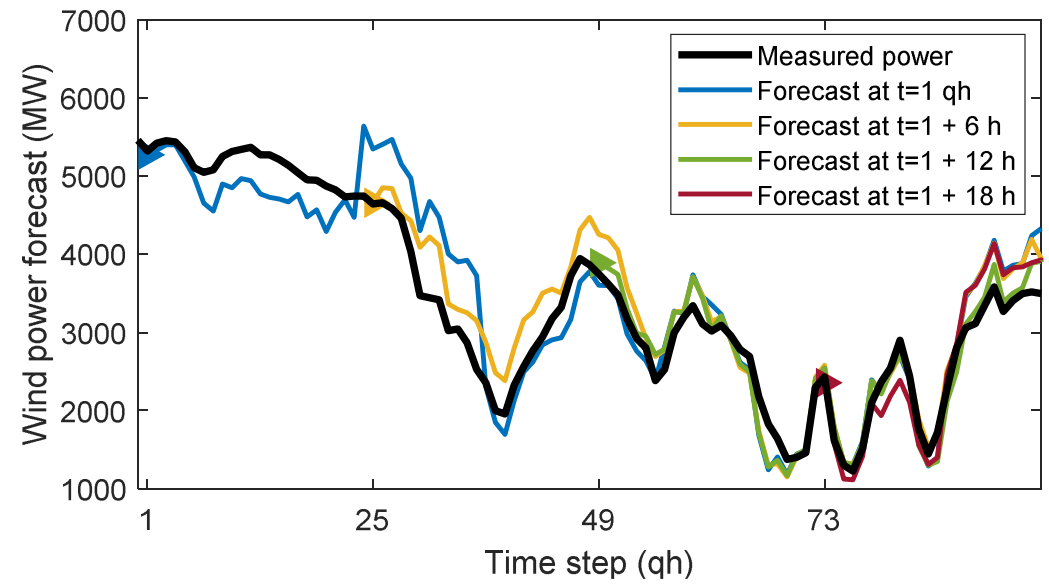

Fig. 2. Superimposing the generated forecast errors on a central wind power forecast (the measured power) yields forecast updates (shown in color). For illustration, an updated forecast is shown in a new color every 6 hours (i.e., $24 \mathrm{qh})$, of which the start is marked with a triangle.

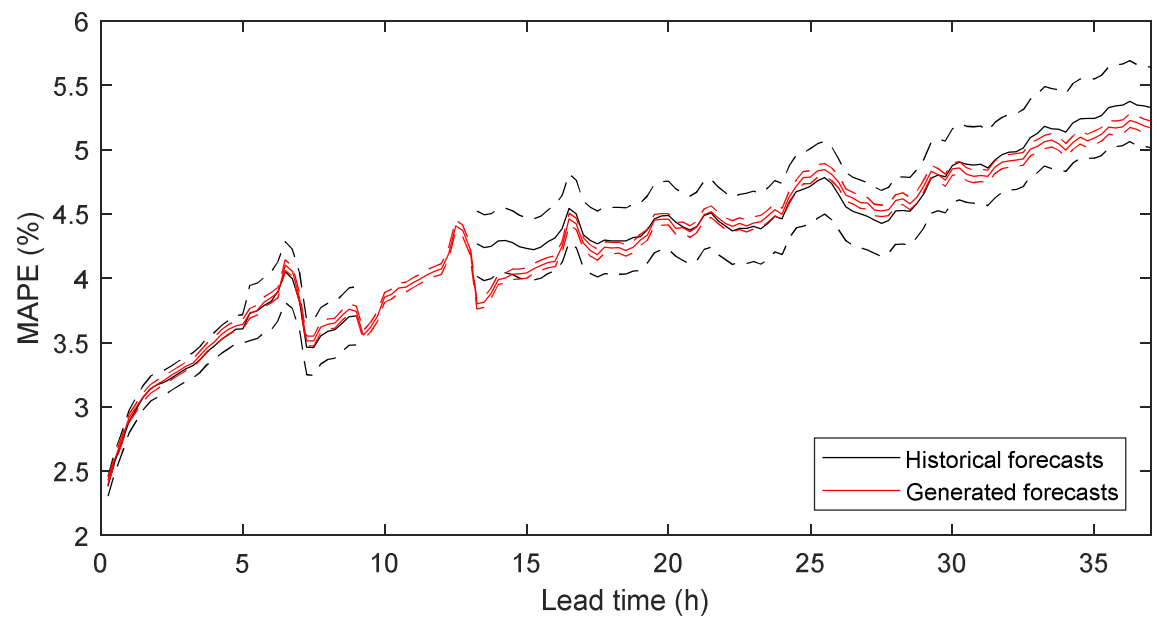

Fig. 3. The MAPE of generated forecasts (10 years) lies within the $95 \%$ confidence intervals of the MAPE of historical forecast data (3 years). Prior to analysis, the time series and forecast errors were normalized with respect to the available wind power capacity. Forecasts with lead times from 1 to $9 \mathrm{~h}$ and 13.25 to $37 \mathrm{~h}$ were available in the historical data. For the missing lead times 9.25-13 h, data was copied from the closest range of day ahead lead times. 


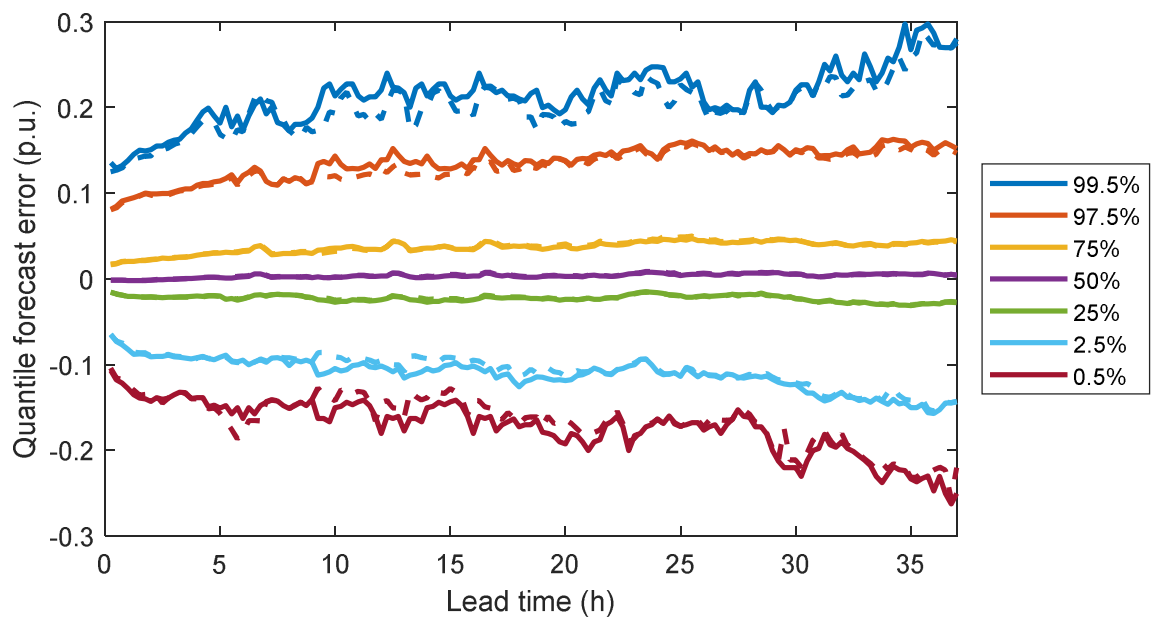

Fig. 4. The quantiles of the historical forecast errors normalized with respect to the available wind power capacity (solid lines) approximate those of the generated forecast errors (dashed lines).

\subsection{Test System and Monte Carlo approach}

Two small scale fictitious generation portfolios of equal size, inspired by the German system before and after a full nuclear and partial coal phase-out, serve as test systems for our case studies. They will be referred to as the low dynamic (LD) and the high dynamic (HD) system. Table II provides an overview of these portfolios and the technical characteristics of their generation units, taken from $[26,27,28,29,30,31,32,33,34]$. The OCGT units are pooled into one single unit with zero minimum load $\underline{P}$. All generation units can provide spinning reserves, and the OCGT can provide non-spinning reserves. The fuel prices are $€ 1.7 / \mathrm{MWh}_{\text {th }}$ for uranium, $€ 8.3 / \mathrm{MWh}_{\text {th }}$ for coal, $€ 4 / \mathrm{MWh}_{\text {th }}$ for lignite and $€ 27 / \mathrm{MWh}_{\text {th }}$ for gas [35]. The CO2-price is set to $6 € / \mathrm{tCO}_{2}$. The CCGT units have two start-up trajectories, outlined in Table III. The fast start-up mode allows for shorter start-up lead times (SUT), equal to those of state-of-the-art CCGTs [28,29], but its associated start-up cost (STC) has an increased maintenance-related share (compliant with [44]), which increases the total STC by a factor of roughly 1.3. It is assumed that a warm start occurs when the unit has been offline for 8 to 50 hours [36]. A start-up after less than 8 or more than 50 hours offline time will be hot or cold. 


\begin{tabular}{|c|c|c|c|c|c|c|c|c|c|c|}
\hline Type & & $\mathrm{HD}$ & $\begin{array}{l}\text { Unit size } \\
\text { [MW] }\end{array}$ & $\begin{array}{c}\text { Efficiency } \\
{[\%]}\end{array}$ & $\frac{P}{[\% \bar{P}]}$ & $\begin{array}{c}S U \\
{[\% \bar{P} / \mathrm{qh}]}\end{array}$ & $\begin{array}{c}R U / R D \\
{[\% \bar{P} / \mathrm{qh}]}\end{array}$ & $\begin{array}{c}M D T / M U T \\
{[\mathrm{~h}]}\end{array}$ & $\begin{array}{c}S T C^{b} \\
{[€ / \mathrm{MW} / \text { start }]}\end{array}$ & $S U T^{\mathrm{c}}[\mathrm{h}]$ \\
\hline Nuclear & 1 & 0 & 1349 & 33 & 50 & 88 & 75 & $24 / 24$ & $61-69-118$ & $10-10-20$ \\
\hline SPP-lignite & 7 & 5 & 310 & 40 & 43 & 51 & 15 & $24 / 24$ & $135-182-228$ & 6-8-10 \\
\hline SPP-coal & 12 & 9 & 310 & 40 & 43 & 54 & 23 & $6 / 6$ & $135-182-228$ & $3-6-10$ \\
\hline SPP-oil & 1 & 1 & 138 & 40 & 43 & 48 & 9 & $5 / 5$ & $135-182-228$ & $3-6-10$ \\
\hline SPP-gas & 4 & 4 & 108 & 40 & 43 & 48 & 9 & $5 / 5$ & $135-182-228$ & $3-6-10$ \\
\hline CCGT-small & 5 & 11 & 57 & 58 & 35 & 58 & 45 & $2 / 4$ & 56-91-139 & $1.5-2.5-4$ \\
\hline CCGT-large & 3 & 8 & 406 & 58 & 35 & 58 & 45 & $2 / 4$ & 56-91-139 & $1.5-2.5-4$ \\
\hline OCGT & 1 & 1 & $480-960$ & 35 & 0 & 100 & 100 & $1 / 1$ & 0 & 0.25 \\
\hline
\end{tabular}

a Aggregated OCGT capacity in LD and HD portfolio.

b Start-up costs for hot, warm and cold starts are taken from [26,34]. Start-up cost are expressed per start and per MW of installed capacity.

c Standard start-up times from notification to minimum load for hot, warm and cold starts are given.

Table II: Low dynamic (LD) and high dynamic (HD) thermal generation portfolios (SPP: steam power plant)

\begin{tabular}{c|cc} 
Start-up mode m & $S T C[€ / \mathrm{MW} / \mathrm{start}]$ & $S U T[\mathrm{~h}]$ \\
\hline Standard & $56.2-91-138.8$ & $1.5-2.5-4$ \\
Fast & $71.2-115.3-175.9$ & $0.5-1-2$
\end{tabular}

Table III: Properties of CCGT start-up modes

Power plant generation is scheduled for one week in April 2016, thereby reducing the computational burden. Quarter-hourly measured wind and solar power output, as well as load time series from the year 2016 are taken from Elia [37]. The wind and solar power output (and capacities) were rescaled (keeping the original ratio between them) such that the total available wind and solar energy in the considered period amounted to $30 \%$ or $60 \%$ (in the high vRES case) of the total electricity demand (1.64 TWh), which corresponds to a solar and wind power capacity of $10.6 \mathrm{GW}$ and $7 \mathrm{GW}$. The upward and downward reserve requirements are split into ten reserve levels. Cogeneration, biomass and hydro power time series of 2013 for Belgium were used as reported by ENTSO-E [38]. Renewables curtailment is assumed to be free and the socio-economic cost of load shedding is assumed to be $10,000 € / \mathrm{MWh}$. Transmission network constraints are not considered.

A Monte Carlo approach is taken to obtain statistically significant cost estimates and reliable insights into the scheduling decisions. Indeed, some wind power forecast update scenarios may trigger the deployment of expensive measures, such as the activation of OCGT peakers or load curtailment, while others pose no such challenges. Therefore, for each case, the considered week is optimized with 210 different forecast update scenarios, yielding 210 UC/ED schedules of which the expected values are reported.

The MIP optimality tolerance is set at $1 \%$. The UC simulations for the 210 scenarios were run in parallel 
on the Genius High Performance Computing cluster of the KU Leuven, using four $2.3 \mathrm{GHz}$ machines with 36 cores and 192 GB of RAM each. Depending on the market clearing frequency and the portfolio, computation times ranged from 11 hours (for an hourly market clearing frequency) to 30 minutes (for a 12-hourly market clearing frequency).

\section{Results and discussion}

In this section, we analyze the impact of the market clearing frequency and the marginal production cost of the OCGT peakers on the operating reserves provision, the use of the start-up flexibility of the CCGT units and the total operating cost, summarized in Table IV. Comparing the reference cases ("Ref.") enabling one ("1 SUM") or multiple start-up modes ("2 SUM") for CCGTs to a case where start-up lead times are not taken into account ("No SU lead times") shows that considering start-up lead times more adequately reveals the impact of more frequent market clearings on the total operational costs. As we will illustrate below, the operating cost savings are driven by the complex interaction between the market clearing and forecast update frequency, operating reserve requirements and start-up decisions of flexible units. This interaction is intensified by an increased share of VRES, but less pronounced in a more dynamic power system, as illustrated by the low-dynamic (“LD”) and high-dynamic (“HD”) power plant portfolios considered in our sensitivity analysis.

\begin{tabular}{|c|c|c|c|c|c|}
\hline \multirow{2}{*}{ Case studya } & \multicolumn{5}{|c|}{ Market clearing frequency $(\mathrm{h})$} \\
\hline & 1 & $3^{e}$ & $6^{e}$ & $12^{\mathrm{e}}$ & $24^{\mathrm{e}}$ \\
\hline Ref. $^{b}-2$ SUM $^{c}$ & 100 & +0.40 & +0.81 & +0.51 & +1.89 \\
\hline Ref. $^{b}-1$ SUMd & 100 & +0.08 & +0.40 & +0.26 & +1.61 \\
\hline Ref. $^{b}-$ No SU lead times & 100 & +0.13 & +0.35 & +0.46 & +1.67 \\
\hline LD VRES $60 \%-2$ SUMc & 100 & +0.10 & +1.00 & +1.31 & +6.87 \\
\hline HD VRES $60 \%-2$ SUM $^{c}$ & 100 & +0.84 & +1.06 & +1.76 & +5.10 \\
\hline
\end{tabular}

a The marginal cost of OCGT peakers equals $1000 € / \mathrm{MWh}$ in all listed cases

b The LD system with $30 \%$ VRES

${ }^{c}$ A standard and fast start-up mode (SUM) is enabled for CCGTs

d Only the standard start-up mode is enabled

e Percent change relative to hourly market clearing

Table IV: Total operational cost results, normalized relative to the total operating cost assuming an hourly market clearing frequency (\%)

In what follows, we first discuss the impact of the market clearing frequency and the peakers' marginal production cost on the operating costs, operating reserve provision and use of the start-up flexibility of CCGT units in our reference case. Note that that the peakers' operating cost may be interpreted as including their long-run marginal costs, i.e., including scarcity rent or as a proxy for the cost of (emergency) demand response. Subsequently, we isolate the impact of the start-up flexibility of these CCGT units on 
the total operating cost in Section 3.2. In Section 3.3, the impact of the power system portfolio and the share of VRES on these interactions are discussed.

\subsection{Market clearing frequency \& marginal cost of OCGT units}

Fig. 5(a) shows the total operational costs with various peakers' marginal production costs and market clearing frequencies. The error bars represent the 95\% confidence intervals around expected total operating cost, calculated as the average of the total operating cost of the $210 \mathrm{UC} / \mathrm{ED}$ schedules (see above). We find that irrespective of the peakers' marginal production costs, the general trend is an expected total operating cost increase with less frequent market clearings, resulting in a cost difference of 1.9-2.3\% between hourly and daily planning. The main driver of this cost increase is the increased wind power uncertainty associated with higher forecasting lead times (Fig. 3). Evidently, the maximum (and average) lead time of the forecasts used for the final planning update (in the commitment stage) increases with the market clearing frequency. As a results, the final planning is made under greater wind power forecast uncertainty, which is reflected in the reserve requirements in the commitment stage and the reserves activation in the balancing stage (Fig. 5(c)).
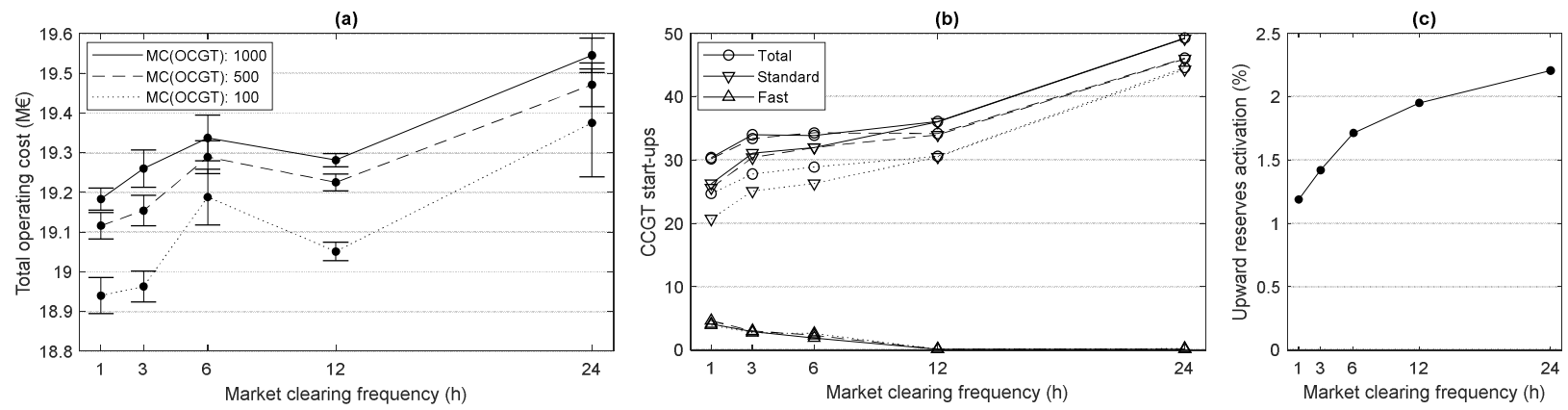

Fig. 5. The total operating costs, with 95\% confidence intervals (error bars), (a) show a general upward trend driven by increased wind power uncertainty for a wide range of the peakers' marginal production costs MC(OCGT) (in $€ / M W h)$. The share of fast starts in the number of CCGT start-ups (b) reaches $20 \%$ with an hourly market clearing frequency. Figure 5(c) shows the upward operating reserves activation in the balancing stage relative to total demand.

Fig. 5(b) shows the total number of CCGT start-ups, split into standard starts and fast starts (Table III). With more frequent market clearings, the need for fast CCGT starts becomes apparent, reaching a share of $20 \%$ at hourly market clearing and peakers' marginal cost of $1000 € / \mathrm{MWh}$. We found that $80 \%$ or more of the fast starts are performed by the small CCGT units, meaning they are more competitive flexibility providers than the large units. This is explained by:

1) their lower start-up costs, which are proportional to the units' rated capacity, and 
2) the mean forecast adjustments that they aim to balance, which are the final improvements of the forecast errors in the commitment stage perceived by hourly market clearing. They can be deduced from the differences between the MAPE values corresponding to lead times in 1-4 qh range and the 58 qh range (see Fig. 3). They are around $0.5 \%$ of the wind power capacity $(7 \mathrm{GW})$, and of the same order as the rated capacity of the small CCGT units.

Increasing the peakers' marginal costs makes CCGT units more competitive as flexibility providers, triggering more start-ups. We observe that a tenfold decrease of the peakers' marginal cost leads to a more than tenfold increase of their planned and final output (not visualized). However, the number of fast starts remains unaffected, while one might expect a decrease of the number of fast CCGT starts as the peakers become more competitive. This means that planning a number of fast CCGT starts is always optimal. Note that while the fast start mode offers a significant advantage to CCGTs in terms of flexibility, the start-up times OCGT peakers are still shorter.

\subsection{Start-up flexibility and lead times of CCGT units}

Figure 6 contrasts the results of three cases: (i) the reference case, enabling the standard and fast startup mode for CCGTs ("2 SUM"), (ii) allowing only the standard start-up mode ("1 SUM"), and (iii) a case where start-up lead times are not taken into account at all when planning ("No SU lead times"). In the last case, the model will always schedule a standard CCGT start, given the lower start-up cost and the absence of start-up lead times. In the balancing stage, however, actually performing these starts may be infeasible (see below). For example, considering hourly market clearings, roughly a quarter of the planned CCGT starts are in reality infeasible. Note that these infeasible starts are allowed in this hypothetical case and the associated cost is that of a standard start.
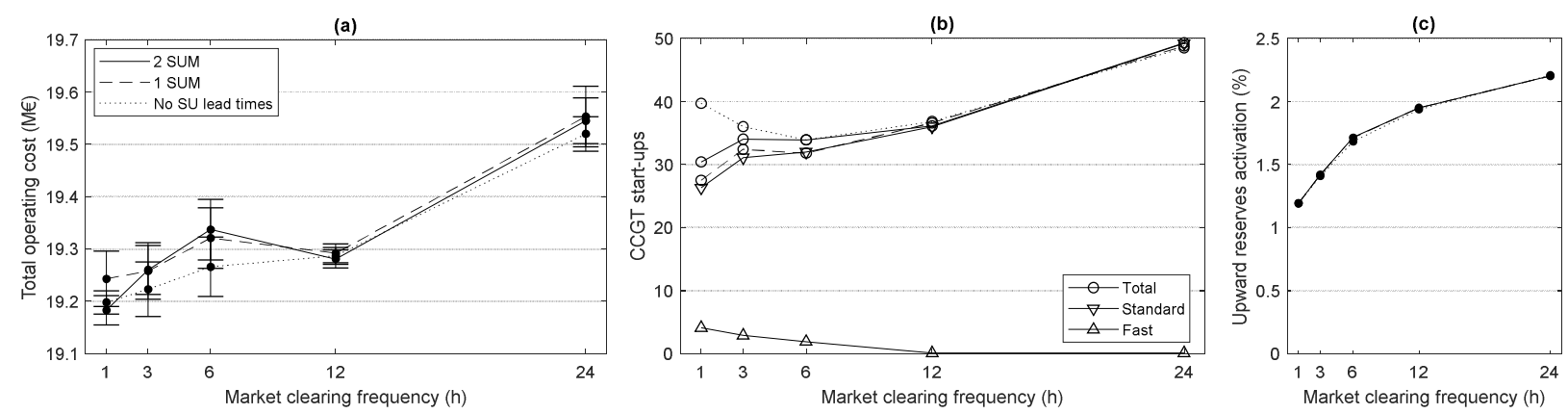

Fig. 6. The general upward trend of the total operating costs (a), driven by increased upward operating reserves activation (c) holds for UC formulations with 2 CCGT start-up modes ("2 SUM"), only one start-up mode ("1 SUM") and without enforcing minimum start-up decision lead times ("No SU lead times"). The start-up lead time restrictions mainly impact the final commitment decisions for hourly up to 6-hourly market clearing frequencies. The need for flexibility offered fast-starting CCGT units (b) is more apparent with more frequent market clearing. 
In all cases, the general trend of the total operating cost increase with lower market clearing frequencies holds. However, when comparing case "2 SUM" and "1 SUM" with case "No SU lead times", we find that the total operating cost for planning frequencies 3 and 6 hours are reduced if we do not account for startup lead times. Imposing start-up lead time restrictions increases the expected operating costs for these market clearing frequencies, as the final commitment decisions are impacted by those restrictions. This causes the increase in operating costs when moving from 12-hourly to 6-hourly market clearing. Indeed, with 6-hourly market clearing, the majority of the final commitment decisions are subject to lead time restrictions because the length of the commitment stage no longer exceeds the (i) the start-up lead times of the CCGT units and (ii) the hot and warm start-up lead times of the SPPs. To maintain the balance between demand and supply in real-time, one must dispatch more OCGT units, which entails a higher operating cost. For market clearing frequencies of 12 or 24 hours, the impact of higher wind power forecast uncertainty (due to longer lead times on the forecasts) dominates.

When contrasting cases " 2 SUM" and " 1 SUM", we observe that the value of the flexibility provided by fast CCGT starts is most apparent with hourly market clearings. In this case, fast starting CCGT units partly replace output of expensive peakers and avoid costly emergency measures such as load curtailment.

In summary, the need for flexibility offered by fast-starting CCGT units and OCGT peakers is more apparent with more frequent market clearings. However, one should take caution when changing the market clearing frequency, as adjusting the production schedule to the most recent vRES forecast update may in some cases bias the decisions towards postponing or cancelling start-ups, leading to more expensive balancing measures and higher operational costs in real time.

\subsection{Share of vRES and Portfolio Flexibility}

In Fig. 7, the results for the reference case are contrasted with the results for both the low dynamic and the high dynamic system with $60 \%$ vRES. First, a more significant operating cost increase with less frequent market clearings is observed when the share of available vRES doubles. With daily market clearings, this cost increase is more limited for the high dynamic portfolio (5.1\%) than for the low dynamic portfolio (6.9\%). Note, however, that the total operational costs for the high dynamic system exceed those for the low dynamic system by roughly $28 \%$ in absolute terms because of the transition from nuclear coal-fired power plants to the more expensive gas-fired power plants. Second, the cost reduction when moving from 6-hourly to 12 -hourly market clearing is absent for the $60 \%$ VRES cases. This is explained by the steeper increase of operating reserve requirements, which forces more spinning reserve providers to stay online, 
which in turn dominates a potential decision bias towards postponing or cancelling start-ups. Together with the steeper increase of reserves activation (which also doubles, see Fig. 7(c)), its impact dominates the cost reductions associated with fewer start-up restrictions (see above). Third, doubling the share of VRES increases the number of CCGT start-ups, especially in the high dynamic system (Fig. 7(b)). Evidently, the greater share of CCGT capacity in the portfolio is the reason for this increase.
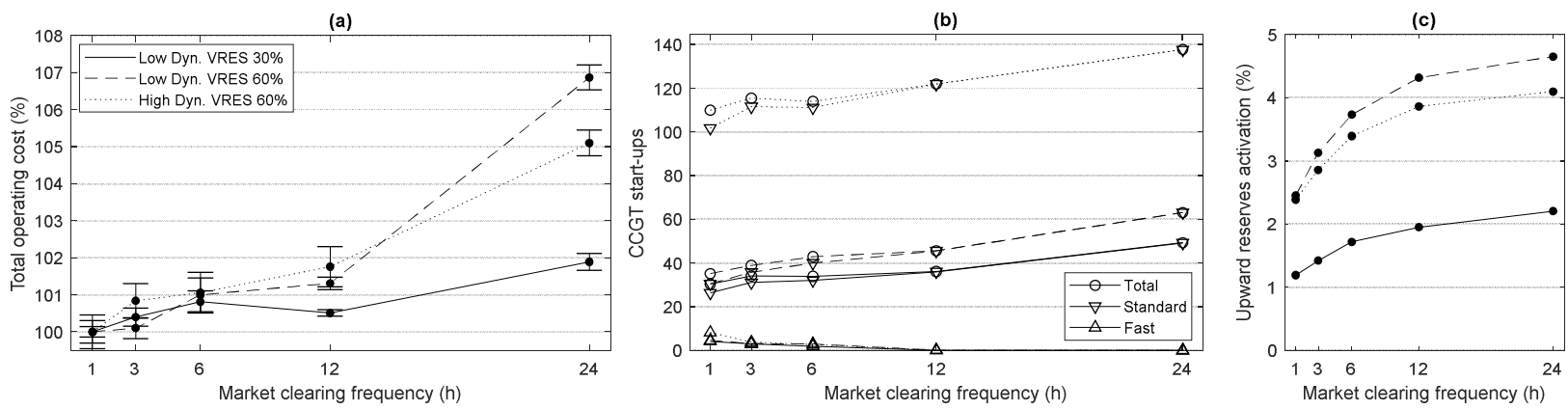

Fig. 7. The total operating costs normalized to the results for hourly market clearing (a) increase steeper for the $60 \%$ vRES cases (both with the low dynamic and the high dynamic system), than for the $30 \%$ vRES reference case, since the upward operating reserves activation is doubled (c). Doubling the share of vRES increases the number of CCGT start-ups, especially in the high dynamic system (b).

In summary, in high vRES scenarios, dynamic electricity generation portfolios with a high share of inflexible capacity and relatively few gas-fired power plants could benefit most from a shift towards sub-daily planning.

\section{Conclusion}

The large-scale introduction of intermittent and limitedly predictable renewable energy sources drives the need for more dynamic power system operation. While previous work has mainly focused on ramping capabilities of generation units, this paper focusses on the interaction between start-up decision lead times, operating reserve requirements and total operating costs in a market environment. Furthermore, we consider shortened start-up procedures of CCGT units at the expense of increased maintenance on the asset.

To this end, we leverage a deterministic unit commitment formulation with probabilistic reserve constraints that enables multiple start-up modes, and apply it in a rolling horizon approach that aims to capture the functioning of joint energy-operating reserve and balancing markets. A dedicated, data-driven tool is deployed to generate wind power forecasts that mimic historical forecasts. In particular, the generated forecasts reflect the correlation of point forecast errors between historically observed wind power forecast updates - a critical feature when adjusting scheduling decisions based on these updates. 
Results of a case study revealed that for the reference test system, the total operational cost increase with less frequent planning, leading to a cost difference of $1.9-2.3 \%$ between hourly and daily market clearing frequencies, caused by increased wind power uncertainty with greater forecasting lead times and facilitated by the fast start-up capabilities of CCGT units. However, one should take caution when changing the market clearing frequency, as adjusting the generation schedule to the most recent wind power forecast update may in some cases bias the decisions towards postponing or cancelling start-ups, ultimately leading to higher operational costs. High vRES systems with generation portfolios with a high share of inflexible capacity (relatively few gas-fired power plants) could benefit most from a shift towards sub-daily planning.

Current intra-day and real-time markets in Europe and the US do allow near continuous trading to adjust unforeseen portfolio imbalances up to an hour or less before delivery, as in the presented model. However, active intra-day trading may be hampered by practical limitations and a lack of liquidity in these intra-day markets, caused by, i.a., the requirement of over-the-counter trading. The bulk of the energy trading hence remains with forward and day-ahead markets. This suggests that the full benefits of more frequent market clearings, as calculated in our case study, are not yet attained. Our results encourage independent system operators (ISO) to optimize their UC decisions taking into account the uncertainty in their system and the start-up lead time restrictions when clearing the market.

In terms of future work, considering multiple sources of uncertainty and including demand response, energy storage and reserve sharing in interconnected power systems may increase the added value of the presented case studies. This work may also be strengthened by taking into account the most recent forecast, as well as previous forecast updates when adjusting the UC schedule.

\section{References}

[1] A. Tuohy, P. Meibom, E. Denny, M. O'Malley, "Unit commitment for systems with significant wind penetration", IEEE Trans. Power Syst., vol. 24, no. 2, pp. 592-601, May 2009.

[2] K. Bruninx, E. Delarue, "A statistical description of the error on wind power forecasts for probabilistic reserve sizing", IEEE Trans. Sustain. Energy, vol. 5, no. 3, pp. 995-1002, Jul. 2014.

[3] J. M. Arroyo and A. J. Conejo, "Modeling of start-up and shut-down power trajectories of thermal units", IEEE Trans. Power Syst., vol. 19, no. 3, pp. 1562-1568, Aug. 2004.

[4] S. Stoft, Power Systems Economics: Designing Markets for Electricity, IEEE Press, John Wiley and Sons, 2002.

[5] J. Boucher, Y. Smeers, "Alternative models of restructured electricity systems part 1: No market power", Oper. Res., vol. 49, no. 6, pp. 821-838, 2001. 
[6] C. Metzler, B. F. Hobbs, J. S. Pang, "Nash-Cournot equilibria in power markets on a linearized DC network with arbitrage: Formulations and properties", Netw. Spatial Econ., vol. 3, no. 2, pp. 123-150, 2003.

[7] M. Hermans, K. Bruninx, E. Delarue, "Impact of CCGT Start-Up Flexibility and Cycling Costs Toward Renewables Integration", IEEE Trans. Sust. Energy, vol. 9, no. 3, pp. 1468-1476, 2018.

[8] E. Bakirtzis, P. Biskas, D. Labridis, A. Bakirtzis, "Multiple time resolution unit commitment for shortterm operations scheduling under high renewable penetration", IEEE Trans. Power Syst., vol. 29, no. 1, pp. 149-159, Jan. 2014.

[9] E. Ela, M. O'Malley, "Studying the variability and uncertainty impacts of variable generation at multiple timescales", IEEE Trans. Power Syst., vol. 27, no. 3, pp. 1324-1333, Aug. 2012.

[10] B. Ummels, M. Gibescu, E. Pelgrum, W. Kling, A. Brand, "Impacts of wind power on thermal generation unit commitment and dispatch", IEEE Trans. Energy Convers., vol. 22, no. 1, pp. 44-51, Mar. 2007.

[11] R. Barth, H. Brand, P. Meibom, C. Weber, "A stochastic unit commitment model for the evaluation of the impacts of the integration of large amounts of wind power", Proc. 9th Int. Conf. Probabilistic Methods Applied to Power Systems, 2006.

[12] E. Ela, "Advanced unit commitment strategies for the U.S. Eastern Interconnection", Proc. 9th Ann. Int. Workshop on Large-Scale Integration of Wind Power into Power Systems as Well as on Transmission Networks for Offshore Wind Power Plants, 2010.

[13] A. M. Jafari, H. Zareipour, A. Schellenberg, N. Amjady, "The value of intra-day markets in power systems with high wind power penetration", IEEE Trans. Power Syst., vol. 29, no. 3, pp. 1121-1132, May 2014.

[14] P. Meibom, H.V. Larsen, R. Barth, H. Brand, A. Tuohy, E. Ela, "Advanced Unit Commitment Strategies in the United States Eastern Interconnection", National Renewable Energy Laboratory (NREL), 2011.

[15] E. Bakirtzis, C. Simoglou, P. Biskas, D. Labridis, A. Bakirtzis, "Comparison of advanced power system operations models for large-scale renewable integration", Electr. Power Syst. Res., vol. 118, pp. 9099, 2015.

[16] T. Schulze, K. Mckinnon, "The value of stochastic programming in day-ahead and intra-day generation unit commitment", Energy, vol. 101, pp. 592-605, 2016.

[17] K. Bruninx and E. Delarue, "Endogenous probabilistic reserve sizing and allocation in unit commitment models: Cost-effective, reliable and fast", IEEE Trans. Power Syst., vol. 32, no. 4, pp. 2593-2603, Jul. 2017.

[18] K. Bruninx, "Improved Modeling of Unit Commitment Decisions Under Uncertainty", Ph.D. dissertation, pp. 150-151, KU Leuven, 2016.

[19] K. Bruninx, E. Delarue, "Scenario reduction techniques and solution stability for stochastic unit commitment problems", Proc. IEEE Int. Energy Conf., pp. 1-7, Apr. 2016.

[20] A. Papavasiliou, S. Oren, and B. Rountree, "Applying High Performance Computing to TransmissionConstrained Stochastic Unit Commitment for Renewable Energy Integration", IEEE Trans. Power Syst., vol. 30, no. 3, pp. 1109-1120, May 2015.

[21] M. Hermans, E. Delarue. "Modeling start-up modes and corresponding cycling costs in the unit commitment problem", IEEE PES PowerTech Conf., Manchester, June 18-22, 2017.

[22] K. Van den Bergh, K. Bruninx, E. Delarue, W. D’haeseleer, "LUSYM: A unit commitment model formulated as a mixed-integer linear program", Nov. 2016, [online] Available: https://www.mech.kuleuven.be/en/tme/research/energy_environment/Pdf/wpen2014-07-2.pdf.

[23] FERC Order No. 764. [Online]. Available at: https://www.ferc.gov/whats-new/commmeet/2012/062112/E-3.pdf 
[24] G. Morales-Espana, J. Latorre, and A. Ramos, "Tight and compact MILP formulation of start-up and shut-down ramping in unit commitment", IEEE Trans. Power Syst., vol. 28, no. 2, pp. 1288-1296, May 2013.

[25] [dataset] Elia, 2019. Grid Data. Available at 〈http://www.elia.be/en/grid-data〉.

[26] A. Schröder, F. Kunz, J. Meiss et al., "Data documentation-Current and prospective costs of electricity generation until 2050", 2013.

[27] D. Lunn and D. Roberts, "Technical assessment of the operation of coal \& gas fired plants", Parsons Brinckerhoff, 2014.

[28] Agora Energiewende, "Flexibility in thermal power plants", p. 116, 2017.

[29] J. Keppler and M. Commetto, "Nuclear Energy and Renewables System Effects in Low-carbon Electricity Systems", NEA, 2012.

[30] C. Ruchti, H. Olia, K. Franitza, A. Ehrsam, W. Bauver, "Combined Cycle Power Plants as Ideal Solution to Balance Grid Fluctuations-Fast Start-up Capabilities", 43th Colloquium of Power Plant Technology Dresden Germany, Sep. 2011.

[31] H. Emberger, D. Hofmann, C. Kolk, "Economic Evaluation of Cycling Plants-An Approach to Show the Value of Operational Flexibility", Power-Gen Europe. 2006.

[32] L. Balling, "Flexible future for combined cycle", Mod Power Syst., vol. 30, pp. 61-63, Dec. 2010.

[33] H. Chandler, "Harnessing Variable Renewables: A Guide to the Balancing Challenge", IEA, May 2011.

[34] Intertek APTECH, "Power Plant Cycling Costs", Nov. 2012.

[35] K. Van den Bergh, "Unit commitment model of the European electricity generation system", Working Paper, 2015.

[36] K. Albert, O. Apelt, G. Bär, and H. Koglin, "Elektrischer Eigenbedarf: Energietechnik in Kraftwerken und Industrie", VDE-Verlag, 1993.

[37] [dataset] Elia, 2017. Grid Data. Available at 〈http://www.elia.be/en/grid-data〉.

[38] [dataset] ENTSO-E, 2019. Statistical Database. Available at 〈www.entsoe.eu/data/dataportal〉.

[39] S. Córdova, H. Rudnick, Á. Lorca and V. Martínez, "An Efficient Forecasting-Optimization Scheme for the Intraday Unit Commitment Process Under Significant Wind and Solar Power", IEEE Trans. Sustain. Energy, vol. 9, no. 4, pp. 1899-1909, Oct. 2018.

[40] P. Pinson, H. Madsen, H. A. Nielsen, G. Papaefthymiou, B. Klöckl, "From probabilistic forecasts to statistical scenarios of short-term wind power production", Wind Energy, vol. 12, no. 1, pp. 51-62, Jan. 2009.

[41] X. Ma, Y. Sun and H. Fang, "Scenario Generation of Wind Power Based on Statistical Uncertainty and Variability", in IEEE Trans. Sustain. Energy, vol. 4, no. 4, pp. 894-904, Oct. 2013.

[42] G. Morales-España, C. M. Correa-Posada, and A. Ramos, "Tight and compact MIP formulation of configuration-based combined-cycle units", IEEE Trans. Power Syst., vol. 31, no. 2, pp. 1350-1359, 2015.

[43] S. Wogrin, D. Galbally, and A. Ramos, "CCGT unit commitment model with first-principle formulation of cycling costs due to fatigue damage", Energy, vol. 113, pp. 227-247, 2016.

[44] J. Janawitz, J. Masso, C. Childs, "Heavy-duty gas turbine op- erating and maintenance considerations", GE Power \& Water, GER-3620M (02/15), 2015.

[45] M. Hermans, K. Bruninx, K. Van den Bergh, K. Poncelet, E. Delarue. "On the Temporal Granularity of Joint Energy-Reserve Markets in a high-RES system", Working paper, KU Leuven.

[46] H. Holttinen, M. Milligan, E. Ela, N. Menemenlis, J. Dobschinski, B. Rawn, R. J. Bessa, D. Flynn, E. Gomez-Lazaro, and N. K. Detlefsen, "Methodologies to determine operating reserves due to increased wind power," IEEE Transactions on Sustainable Energy, vol. 3, pp. 713-723, Oct 2012.

[47] R. Widiss and K. Porter, "Review of variable generation forecasting in the west: July 2013 - march 2014," tech. rep., National Renewable Energy Laboratory (NREL), 32014. 
[48] A. Tuohy, J. Zack, S. E. Haupt, J. Sharp, M. Ahlstrom, S. Dise, E. Grimit, C. Mohrlen, M. Lange, M. G. Casado, J. Black, M. Marquis, and C. Collier, "Solar forecasting: Methods, challenges, and performance," IEEE Power and Energy Magazine, vol. 13, pp. 50-59, Nov 2015.

[49] M. G. Prina, L. Fanali, G. Manzolini, D. Moser, and W. Sparber, "Incorporating combined cycle gas turbine flexibility constraints and additional costs into the EPLANopt model: The Italian case study," Energy, vol. 160, pp. 33-43, 2018.

[50] G. Garegnani, M. G. Prina, R. Vaccaro, M. Cozzini, U. F. Oberegger, and D. Moser, "EPLANopt: EnergyPLAN optimization library," 2016, Available at 〈https://gitlab.inf.unibz.it/URS/EPLANopt〉. 